\title{
Computations of the Hill Functions of Higher Order
}

\author{
By A. J. Jerri
}

\begin{abstract}
In this note, we express the hill function $\phi_{n}(x)$ of an order $n$ as a Fourier cosine series which is of simple form that allows proving the function's basic properties. For the hill functions of higher order $(15<n<50)$ the form of the coefficients makes the series "essentially" self-truncating. For such high order hill functions, this truncated series (with thirty terms) computes the hill function with the same accuracy as the method of Legendre polynomials with local coordinates, but without the latter required $n^{2}$ coefficients which are to be computed in advance. The preliminary time analysis indicates that the time for the two methods starts to be the same at $n \sim 15$, changes slightly for the cosine series for $n>15$ and varies roughly as $n^{3}$ for the localized Legendre polynomial method. In comparison with the most recent efficient methods which require a storage of order $n$, this note's method required a storage of the order 25-40 for $n<60$, executed with almost the same speed and accuracy and stayed stable as long as the above methods did.
\end{abstract}

I. Introduction. Consider the function

$$
F(\omega)=\frac{1}{2 \pi} \int_{-\infty}^{\infty} f(t) e^{-i \omega t} d d t
$$

and its Fourier transform

$$
f(t)=\int_{-\infty}^{\infty} F(\omega) e^{i \omega t} d \omega .
$$

We note that the Fourier transform of the gate function (the hill function of order one),

$$
\phi_{1}(a, \omega)= \begin{cases}1, & |\omega| \leqslant a \\ 0, & |\omega|>a\end{cases}
$$

is $(2 \sin a t) / t$.

The hill function $\phi_{R+1}(a(R+1), \omega)$, of order $R+1$, is defined as the $R$ th fold Fourier convolution of $\phi_{1}(a, \omega)$; and hence, it is the Fourier transform of [(2 sin at $) / t]^{R+1}$. The exact explicit form of $\phi_{R+1}(a(R+1), \omega)$ is given in [1] for each of the $(R+1) / 2$ and $(R+2) / 2$ subintervals of $[0, a(R+1)]$ for $R$ odd and $R$ even, respectively. The hill function $\phi_{R+1}(a(R+1), \omega)$ is an even function in $\omega$, a polynomial of degree $R$ with continuous derivatives up to $R-1$ and vanishes outside the interval $[-a(R+1), a(R+1)]$. Unless otherwise indicated, we will write $\phi_{R+1}(\omega)$ for $\phi_{R+1}(a(R+1), \omega)$.

The hill functions of higher order have proved important in the finite element method [2] of approximating the solutions by a finite sum. For such high orders the computations of the explicit form [1] show an extreme instability, so Legendre

Received August 28, 1975 ; revised July 7, 1976.

AMS (MOS) subject classifications (1970). Primary 41 A15, 42 A16. 
polynomial expansions [3] , with localized coordinates for each of the $R+1$ subintervals, and other more efficient methods [4] , [5] were recently employed to eliminate this instability. The method in [3] is an accurate finite sum but, for large $R$, it does put a constraint on the storage locations, required for its precomputed $(R+1)^{2}$ coefficients. This is not the case for the most recent methods [4] , [5] where the required storage is of order $R+1$. Also, neither the exact explicit form [1] nor the localized Legendre polynomial [3] form seem to be suitable for proving some basic properties of the hill function which we plan to illustrate.

In the next section, we will express $\phi_{R+1}(\omega)$ as a Fourier cosine series on the whole integral $[-a(R+1), a(R+1)]$ with a simple form for the coefficients that makes the series "essentially" self-truncating. In the last section, we will use this series to prove the basic property of the hill function, that the average of $\phi_{R+1}(\omega)$ over the whole interval $[-a(R+1), a(R+1)]$ is equal to the average of its $R+1$ midsubinterval values. Such property sheds a light on the importance of the hill functions, since it simply states that one point weighted by the hill function $\phi_{R+1}$ of order $R+1$ is equivalent to $R+1$ points weighted by the hill function $\phi_{1}$ of order one.

II. Computations of the Hill Functions. The hill function $\phi_{R+1}(\omega)$ is expressed as a Fourier cosine series,

$$
\begin{aligned}
\phi_{R+1}(\omega)=\frac{a_{0}}{2}+\frac{1}{a(R+1)} \sum_{n=1}^{\infty}\left[\frac{2 \sin n \pi /(R+1)}{n \pi / a(R+1)}\right]^{R+1} \cos \frac{n \pi \omega}{a(R+1)}, \\
-a(R+1)<\omega<a(R+1),
\end{aligned}
$$

where $a_{0}=(2 a)^{R+1} / a(R+1)=2 a \bar{\phi}_{R+1}$ and $\bar{\phi}_{R+1}$ is the average value of $\phi_{R+1}(\omega)$ over the interval $[-a(R+1), a(R+1)]$, with value of $(2 a)^{R} /(R+1)$. In (4) we note the simple form of the coefficients and their advantage in making the series a selftruncating one for large $R$. We may also mention that this property of the hill function's Fourier coefficients has been used [6] indirectly to make the cardinal (sampling) series, in communication theory, a self-truncating one.

For large $R(R \geqslant 10)$ the $N$ th $(N \sim 30)$ partial sum of $(4)$ gives as accurate an approximation of the hill function as that of the Legendre polynomial expansion [3] with local coordinates. Such $N$ term computations may be compared with $R+1$ terms for each of the $(R+1) / 2$ subintervals ( $R$ odd) a total of $(R+1)^{2} / 2$ terms, required by the localized Legendre polynomials method to describe $\phi_{R+1}(\omega)$ on the whole interval. This is in addition to the latter method storage constraint for the $(R+1)^{2}$ coefficients that have to be computed in advance. The preliminary computations done on IBM 360/44-S show that for $R=10$, with the same accuracy, the exact explicit form [1] takes the least time followed by the Legendre method. As $R$ reached 15 , the explicit form suffered from instability while the Legendre and the cosine method computations took approximately the same time. As $R$ increased to 20, the cosine series time $(N=30)$ changed very slightly while the time for the localized Legendre series varied roughly with $R^{3}$. For $R>20$ we could not compare our 
results to the Legendre expansions one since the latter coefficients storage requirement exceeded the storage capacity of the IBM 360/44-S. This is an obvious disadvantage to the localized Legendre method when we consider the availability of only fair-sized machines. However, this is not the case for most recent efficient methods [4] , [5] where our preliminary computations show that for $R<60$, with double precision, such methods require a storage of the order of $R+1$ coefficients compared to $N=25-40$ coefficients for the note's method. Also for $R<60$, our method executes with about the same speed, almost the same accuracy with trailing off at the ends, and stays stable as long as the method in [4] does.

III. A Basic Property of the Hill Functions. Here we will use the Fourier cosine series (4) of the hill function $\phi_{R+1}(\omega)$ to show that $\bar{\phi}_{R+1}$, the average value of $\phi_{R+1}(\omega)$ over the interval $[-a(R+1), a(R+1)]$, is equal to the average of its $R+1$ values at the middle of each of the $R+1$ equal subintervals of $[-a(R+1), a(R+1)]$. We will first consider the case of $R$ odd where $\phi_{R+1}(\omega)$ is defined on $R+1=2 m$ equal subintervals. The case of even $R$ will follow in the same way with $R+1=$ $2 m+1$ equal subintervals. Let $\rho_{k}(\omega)$ be the part of $\phi_{R+1}(\omega)$ defined on the two symmetric subintervals $(-2(k+1) a,-2 k a)$ and $(2 k a, 2(k+1) a) . \rho_{k}(\mp(2 k+1) a)$ are the values of the hill function $\phi_{R+1}(\omega)$ at the middle of two such symmetric $k$ th subintervals. The sum of these $2 m=R+1$ midsubinterval values is obtained from (4) as

$$
\begin{aligned}
\sum_{k=0}^{(R-1) / 2} & \rho_{k}(\mp(2 k+1) a) \\
= & \frac{(R+1) a_{0}}{2}+\frac{1}{a(R+1)} \sum_{n=0}^{\infty}\left[\frac{2 \sin n \pi /(R+1)}{n \pi / a(R+1)}\right]^{R+1} \\
& \cdot 2 \sum_{k=0}^{(R-1) / 2} \cos \frac{n \pi(2 k+1)}{R+1} .
\end{aligned}
$$

But

$$
\sum_{k=0}^{(R-1) / 2} \cos (2 k+1) \alpha=\frac{\sin (R+1) \alpha}{2 \sin \alpha}
$$

which makes the double series vanish since the finite sum over $k$ vanishes when $n /(R+1)$ is not integer, and every term in the infinite series vanishes when $n /(R+1)$ is an integer. Hence

$$
\frac{1}{R+1} \sum_{k=0}^{(R-1) / 2} \rho_{k}(\mp(2 k+1) a)=\bar{\phi}_{R+1}
$$

is the desired result. To show (6) for even $R$ the same steps would be followed except that we now consider $R+1=2 m+1$ equal subintervals. 
1. V. A. DITKIN \& A. P. PRUDNIKOV, Integral Transforms and Operational Calculus, Fizmatgiz, Moscow, 1961; English transl., Pergamon Press, New York, 1965, pp. 177-178. MR 33 \#609.

2. I. BABUŠKA, "Approximation by Hill functions," Comment. Math. Univ. Carolinae, v. 11, 1970, pp. 787-811. MR 45 \#1396.

3. J. SEGETHOVÁ, "Numerical construction of the Hill functions," SIAM J. Numer. Anal., v. 9, 1972, pp. 199-204. MR 46 \#682.

4. C. de BOOR, "On calculating with B-splines," J. Approximation Theory, v. 6, 1972, pp. 50-62. MR 49 \#3381.

5. C. de BOOR, Package for Calculating with B-Splines, Tech. Report \#1333, Math. Research Center, Univ. of Wisconsin, Madison, 1973.

6. H. D. HELMS \& J. B. THOMAS, "Truncation error of sampling-theorem expansions," Proc. IRE, v. 50, 1962, pp. 179-184. MR 26 \#5707. 